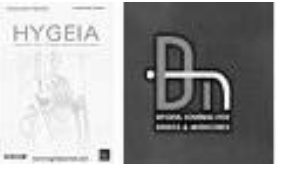

\title{
Propolis non-volatile constituents: A Review
}

\author{
Konstantinos M. Kasiotis \\ Benaki Phytopathological Institute, Laboratory of Pesticides Toxicology, 8 St. Delta Street, Athens, Kifissia 14561, Greece \\ Article history: Received: 8 March 2013, revised: 10 March 2014, accepted: 15 March 2014, Available online: 3 April 2014
}

\begin{abstract}
Plan: This study includes the characteristics and variation of non volatile constituents in propolis samples collected from different regions of the world. The review covers the research activities after 2010 on propolis constituents.

Outcome: Propolis constitutes a complex resinous material collected by honeybees serving as a defense system for their hives. Apart from this evident natural use, propolis is marketed by the pharmaceutical industry due to its claimed beneficial effects on human health such as antioxidant, antibacterial, antiviral and anticancer activities. These properties are attributed to the chemical compounds that are found in propolis mainly phenolic compounds and terpenes. In this review article the analysis of non-volatile compounds of propolis performed by high performance liquid chromatography (HPLC) coupled with various detectors, will be presented. Future trends and further insights are quoted and commented.
\end{abstract}

Key words: Non volatile constituents, Propolis.

\section{INTRODUCTION}

Propolis is a natural product that belongs to the great family of bee products. The word propolis is a complex word originating from two ancient Greek words: pro- standing for "before or in defense" and polis-meaning city. Thus in apiculture its meaning refers to the shield of the hive. Propolis is a sticky, resinous substance, collected from various floral sources that is transformed and used by honeybees to construct and maintain their hives by sealing holes in their honeycombs, smooth out the internal walls and shelter the entrance of the hive from intruders. Trends and development in propolis research have been reviewed by Bankova(Bankova, 2005b). In this view, key point in any research conducted is the chemical variability of propolis attributed to the diversity of its plant origin (Bankova, 2005a).Propolis is a traditional remedy in folk medicine that has been used for centuries in Egypt, Greece and other countries as well. It is known that propolis possesses anti-microbial, anti-oxidative, anti-ulcer and anti-tumor activities and the latter is proved by a plethora of reports on these activities. A very good review article on the biological activity of bee propolis in health and disease was published by Lofty in 2006 (Lofty, 2006), collecting many important works on various medicinal aspects.

For Correspondence: K.Kasiotis@outlook.com

Contact: Tel: 0030-210-8180357, 384 ; Fax: 0030-210-8180223

Hygeia.J.D.Med. Vol.6 (1), April 2014 @ 2014 all rights reserved.

Hygeia journal for drugs and medicines, 2229 3590, 09756221

Rid. D-4713-2014 
A Greek work of 2010 has highlighted the anti-proliferative activity of Greek propolis (Pratsinis et al., 2010). Brazilian propolis has also been reported to suppress angiogenesis by inducing apoptosis in tube-forming endothelial cells (Kunimasa et al., 2011).

Additionally propolis has exhibited significant activity in the field of oral health, a part in which polyphenols contribution was not highlighted. In this regard a good review was published by Varoni et al. (Varoni et al., 2012) regarding the beneficial character of polyphenols included in propolis. Propolis composition comprises of several phytochemicals, including various phenolic compounds such as flavonoids (eg. Chrysin, kaempferol, see Figure 1), cinnamic acid and its derivatives (chlorogenic acid, ferulic and caffeic acid methyl esters). Moreover propolis contains various volatile constituents such as terpenoids (Melliou et al., 2007) however these do not fall within the scope of this review. The plant source of propolis depends on the specific flora at the site of collection.

Figure 1. Some flavonoids found in propolis<smiles>O=c1cc(-c2ccccc2)oc2cc(O)cc(O)c12</smiles><smiles>O=c1c(O)c(-c2ccc(O)cc2)oc2cc(O)cc(O)c12</smiles>

Chrysin

Kaempferol<smiles>O=C1C[C@H](c2ccccc2)Oc2cc(O)cc(O)c21</smiles>

Pinocembrin

Accordingly, the constituents of propolis from different geographical locations may be diverse. European propolis contains flavonoids and cinnamic acid derivatives as the main constituents and principal source are the poplar trees, while Brazilian green propolis contains labdane diterpenes and prenylated phenolic compounds, and Baccharisdra cunculifolia is considered as the main botanical source. Brazilian red propolis originates from plants belonging to genus Dalbergia and contains predominant amount of flavonoids and pterocarpans.

Amongst analytical techniques, the thin layer chromatography (TLC) is still commonly regarded as the basic tool for the identification of natural compounds, and is used to provide the initial characteristic fingerprints of a sample. Furthermore, modern instrumental high performance TLC (HPTLC) is widely applied in quantitative analysis, and two dimensional TLC has also found ground. In depth analysis of propolis is achieved by chromatographic methods, usually liquid and gas chromatography (LC and GC) [indicatively see (Popova et al., 2011; Shi et al., 2012)]. 
LC is used for the analyses of non-volatile constituents predominated by phenolic compounds. GC serves principally for the analyses of volatile constituents or for the analyses of phenolic constituents as well after derivatizing the compounds so as to be amenable for GC. Mass spectrometry (MS) is critical for the identification of structure of compounds. Thus identification of non-volatile compounds is mainly performed by high performance liquid chromatography mass spectrometry(HPLC-MS) analyses and based on the deprotonated molecule $[\mathrm{M}-\mathrm{H}]^{-}$along with the interpretation of its $\mathrm{MS}^{\mathrm{n}}$ fragmentations, since most of works are based on negative ionization mode functioning. However there are reports which use positive mode so in that case $[\mathrm{M}+\mathrm{H}]^{+}$is the pursued ion. Noteworthy is, that prior to any separation is usual to apply a direct infusion of the extract to the mass spectrometer so as to acquire an initial fingerprint which provides valuable information as regards the predominant ions, hypothesizing potential compounds that match to the latter(Aguero et al., 2010). Furthermore, spectrophotometric method has been proposed for the quantification of prenylated flavanones in propolis (Popova et al., 2010).

The purpose of this review is to summarize currently available reports on the chemical composition of propolis, with emphasis on liquid chromatography applications. The review will cover principal works after 2010 on the non-volatile chemical composition of propolis embracing representative works on worldwide scale. Finally probable future insights and trends will be quoted.

\section{MAJOR REPORTS}

\section{Asian works: Thai propolis}

Chemical constituents of Thai propolis were investigated by Athikomkulchai et al.(Athikomkulchai et al., 2013). In this regard Thai propolis was extracted with methanol assisted by sonication and evaporated under reduced pressure to give a methanol extract. A part of the extract was chromatographed on silica gel with a methanol-dichloromethane gradient system to give 16 fractions. These fractions were further investigated by normal and reversed phase preparative TLC and crystallization steps to give known and new compounds which were characterized by extensive nuclear magnetic resonance(NMR) spectroscopic techniques and high resolution mass spectrometry.

In present study, the isolation of a new phenylallylflavanone,(7"S)-8-[1-(4'-hydroxy-3'methoxyphenyl)prop-2-en-1-yl]-(2S) pinocembrin and (E)-cinnamyl-(E)-cinnamylidenate from methanolic extract of Thai propolis was reported. In addition to this, 19 compounds belonging to flavonoids and phenolic esters were isolated and identified. The isolated compounds comprises mainly flavonoids and phenolic esters, which wereclose to those reported from the propolis of temperate regions in Europe, China, Uruguay and Mexico, and whose botanical sources are believed to be poplar trees such as Populus nigra.

\section{Chinese Propolis}

In 2012 Shi et al. investigated fifteen propolis samples collected from different regions of China(Shi et al., 2012). In this regard their phytochemical composition was determined and compared, and anti-inflammatory and radical scavenging properties were investigated. 
Eleven compounds including caffeic, p-coumaric, ferulic, isoferulic, and 3,4-dimethyl caffeic acids, pinobanksin, chrysin, pinocembrin, galangin, pinocembrin 3-acetate and caffeic acid phenyl ethyl ester were quantified using an ultra-high performance liquid chromatography (UHPLC) method in an overall runtime of 15 minutes, and on a per propolis wet basis. Analytical figures of merit apart from linear range and correlation coefficient values are not presented. The data from this study showed that Chinese propolis was rich in phenolic acids, flavonoids and phenolic acid esters. Four flavonoids, chrysin, pinocembrin, galangin, and pinobanksin 3-acetate were the primary constituents in most tested Chinese propolis samples. Additionally 38 compounds were identified by Time of Flight mass spectrometry UHPLC/QTOF-MS. The 15 samples significantly differed in their total phenolic and total flavonoid content, as well as their phytochemical profiles. This study also confirmed that Chinese propolis samples belonged to the poplar-type propolis(Bankova, 2005b).

\section{Indonesian Propolis}

An extract of Indonesian propolis was studied for its chemical composition, biological activity and botanical origin. In order to proceed to chemical compounds identification, the propolis was extracted with chloroform. Purification by repeated column chromatography and preparative TLC one inseparable mixture, one complex mixture and four individual compounds were obtained. The inseparable mixture contained resorcinolic compounds while the complex mixture comprised of prenylflavanones which were identified after derivatization and GC-MS detection. The most abundant category of compounds was propolins known for their biological activity (Shirataki et al., 2001).

\section{South America: Argentinean Propolis}

Aguero et al. published a work on Argentinean propolis phytochemical characterization and evaluation of antifungal activity(Aguero et al., 2010). In that work propolis was methanol extracted and the extracts were subjected to fractionation by column chromatography. Analyses were conducted with HPLC-DAD, while MS and MS/MS data for confirmation purposes were acquired by direct infusion of pure compounds. Then propolis extracts were qualitatively evaluated using HPLC-ESI-MS/MS using a C18 column of the separation of compounds. Limit of Detection \& Quantification (LOD \& LOQ) values are not mentioned although the work seems fully validated. Bioactive compounds identified were one methoxychalcone, one dihydroxychalcone, galangin, pinocembrin and 7-hydroxy-8-methoxy-flavanone. The two chalcones exhibited remarkable antifungal activity as demonstrated by the low minimum inhibitory concentrations near $2 \mu \mathrm{g} / \mathrm{mL}$.

\section{Red propolis from Brazil and Cuba}

The existence of a red colored propolis has been reported in several countries, such as the northeastern coast of Brazil, Mexico, Cuba, Venezuela and China [see references in (Lopez et al., 2014)]. In this context Lopez et al. have reported the characterization of red propolis samples collected from different regions of Brazil and Cuba(Lopez et al., 2014) introducing a fingerprinting-marker approach. To proceed authors choose to initially direct infuse samples in electrospray ionization mass spectrometer functioning in negative ionization mode and deduce the mass spectra fingerprint. 
In this context the data regarding $\mathrm{m} / \mathrm{z}$ values and abundances of $\mathrm{m} / \mathrm{z}$ ions were classified in groups using principal component analysis (PCA) based on the common ions in the fingerprints and previously published results. The extraction of samples was performed with ethanol and a then freezing overnight step was adapted in order to remove the wax. In case of further subjection to UPLC-ESI (-)-MS/MS experiments the solvent was evaporated and the dry extract $(1 \mathrm{mg} / \mathrm{mL}$ ) was passed from a C18 column. Mobile phase consisted of $0.1 \%$ formic acid in water (A) and methanol (B) using a linear gradient. The propolis samples were classified into 3 main groups and characteristics marker ions for each of the three groups could be identified. The Brazilian samples from one state displayed a very abundant market at $501.3 \mathrm{~m} / \mathrm{z}$ which needs further studies to be characterized. In the second group ions specific for formononetin, biochanin A and pinocembrin were identified. For the third group a marker ion at 601.35 was possibly attributed to a benzophenonic molecule.

\section{Yellow Propolis from Cuba}

Yellow propolis samples collected from Cuba were studied for their constituents in a study where GC was also involved(Marquez Hernandez et al., 2010). The study was based on information supplied by NMR, HPLC-DAD and HPLC-ESI-MS data and then a qualiquantitative GC-MS study was developed, especially for aliphatic compounds (triterpenoids). Specifically propolis samples were extracted with methanol, and the evaporated extract along with the IS were derivatized with $\mathrm{N}$-methyl-N-trimethyl silyl trifluoroacetamide. The breakthrough of this work was that for the first time some triterpenes belonging to oleanane, lupine, ursane and lanostane skeletons were reported as major compounds.

\section{African region: Algerian Propolis}

A 2013 work published by Piccinelli et al. was focused on the elucidation of chemical composition of propolis samples collected from north Algeria (Piccinelli et al., 2013). The authors applied an initial fingerprinting using HPLC-DAD which allowed the classification of two main types of Algerian propolis; the one that was rich in polyphenols and the other rich in diterpenes. Their further investigation was pursued with preparative chromatography procedures followed by NMR and mass spectrometry analyses. As regards the polyphenolic Algerian propolis type, major constituents were three caffeic acid derivatives, the flavonols chrysin and apigenin, two flavonols (kaempferol and galangin), and three flavononols (pinobanksin, pinobanskin 3-O-acetate, and pinobanksin 3-(E)-caffeate). The caffeate of pinobanskin until that work has never been reported in propolis samples.

Flavonoids from Algerian propolis have also been reported by Segueni et al.(Segueni et al.). Propolis was collected from the north of Algeria and afforded five flavones: pectolinaringenin, pilosin, ladanein, chrysin, apigenin. The structures were elucidated by spectroscopic analysis, including mass spectrometry, one and two dimension (1D and 2D) NMR. The importance of this paper is highlighted by the identification of pectolinaringenin and ladanein which are reported for the first time in propolis.

In 2014 a work on Algerian propolis was published focusing on the scavenging activity of ethyl acetate extracts and myeloperoxidase (MPO) activity inhibition (Boufadi et al., 2014). 
Fractionation and analyses were performed by preparative chromatography and LC coupled with TOF mass detector respectively. The authors concluded that kaempferol, one of the constituents, seems to play a predominant role to MPO inhibition.

\section{Kenyan Propolis}

Kenyan propolis after initial investigation with TLC and GC-MS, and comparison with other type propolis exhibited a different chemical composition pattern (Petrova et al., 2010). In this context two new arylnapthalene lignans were isolated tetrahydrojusticidin and 6methoxydiphyllin, along with four known phenolic compounds found for the first time in propolis.

\section{Europe and Mediterranean: Italian Propolis}

The composition and anti-oxidant activity of Italian poplar propolis obtained using three harvesting methods and extracted with different solvents (ethanol, acetone, and chloroform) were assessed (Papotti et al., 2012). The highest extraction capacity was displayed by acetone. Phenolic composition was determined using HPLC-MS/MS analysis of diluted -with methanolextracts. Chromatography was performed on a Zorbax Eclipse XDB C18 column. Identification of compounds was conducted by comparing experimental retention time and the mass spectrometry fragmentation patterns with those of pure standard compounds assisted by literature data. LOD and LOQ values were determined by the $\mathrm{S} / \mathrm{N}$ approach. Main compounds detected by all extraction protocols were apigenin, chrysin, galangin, kaempferol, quercetin, naringenin, pinocembrin, pinostrobin, pinobanksin, p-coumaric acid, ferulic acid, caffeic acid, caffeic acid phenethyl ester (CAPE), caffeic acid cinnamyl ester and pinobanksin 3-O-acetate.

Pellati et al. have published a competent chemical analysis of Italian raw propolis samples (Pellati et al., 2013).

Nine samples of propolis located in different regions were collected and frozen at $-20{ }^{\circ} \mathrm{C}$. Then they were finely powdered and extracted following four methods; including maceration, heat reflux extraction, ultrasound-assisted extraction and microwave assisted extraction (MAE). MAE proved the most efficient method. Analyses of samples were performed with HPLC-DAD validated according to ICH guidelines (ICH, 2005).LOD and LOQ values were determined after serial dilutions of a standard solution to reach a signal to noise ratio of 3 and 10 respectively. The authors by using the fused-core $\mathrm{C} 18$ column managed to reduce total analysis time from 92 (when using fully porous column) to $65 \mathrm{~min}$. In the samples analyzed the most abundant flavonoids were chrysin, pinocembrin, galangin, and pinobanskin-3-O-acetate. As regards phenolic acids, caffeic acid, $p$-coumaric acid and ferulic acid were the most profuse ones. For phenolic acid derivatives 3,4-dimethyl caffeic acid, caffeic acid prenyl, benzyl, phenylethyl and cinnamyl esters were the most representative compounds.

\section{Serbian Propolis}

Serbian propolis samples were collected and examined for their chemical composition, and ethanolic extracts were assessed for cytotoxic and anti-oxidative activities (Zizic et al., 2013). Characterization of composition was achieved by HPLC-DAD. LOD and LOQs were determined following the S/N approach. 
As regards the quantitative analyses results caffeic acid, CAPE, ferulic and isoferulic acid were the most abundant compounds from phenolic acids. Regarding flavonoids chrysin, pinocembrin and galangin were detected in higher amounts than other compounds. The authors concluded that these Serbian propolis samples resembled analyzed samples from Bulgaria, Italy and Switzerland (see references therein).

\section{Croatian Propolis}

A good mini-review article on Croatian propolis analyses (and wine, which is not the scope of this review) was published by Medic-Saric et al . The authors focused on propolis production, chemical analysis (spectrophotometry, HPLC, HPTLC, GC/MS, etc.) of flavonoids and phenolic acids, all of which enable authentication and geographical traceability of propolis. This work represents the basis for quality control and regulatory framework for any dietary supplement claiming to have beneficial health effectiveness.

\section{Maltese Propolis}

Popova et al. has published a work on Maltese propolis. In this view ethanol extracts were subjected to: 1) derivatization under basic conditions to afford silylated extracts analyzed by GC-MS and 2) preparative TLC so as to isolate individual compounds and characterize them by NMR. The samples were rich in terpenes and sugars showing resemblance with other Mediterranean propolis samples (see Greek references below). Only samples with high content in terpenyl esters showed antifungal activity.

\section{Greek propolis}

For the Greek propolis phytochemical constituents few reports are published until now. In the first report of 2009 chemical composition, antioxidant activity and in vitro antimicrobial activity of twelve propolis ethanolic extracts from mainland Greece, Greek islands and east Cyprus were determined(Kalogeropoulos et al., 2009). The authors used GC-MS analysis operating in SCAN (total ion current, TIC) or selective ion monitoring mode (SIM) after derivatizing all analytes with Bis-(trimethylsilyl)-trifluoroacetamide (BSTFA).The presence of anthraquinones and terpenes in significant amounts and the low amounts of phenolic acids and their esters, disclosed the similarity in chemical constituents of these samples with propolis of East Mediterranean rather than typical European propolis.

A further insight into the chemical composition of Greek propolis was given by a Greek and Turkish collaboration(Celemli et al., 2013) where comparison with Turkish propolis composition was made. Extraction of frozen ground propolis was achieved with ethanol in a ratio of $1: 3(\mathrm{w} / \mathrm{v})$. The mixture was kept in an incubator at $30^{\circ} \mathrm{C}$ for two weeks. After filtration and evaporation, the dry extract was subjected to derivatization with BTFSA and GC-MS analysis.In this study it was again confirmed that Greek propolis with its high terpene content belongs to the Mediterranean type propolis. The Turkish samples from the coast line of the Aegean Sea are similar to the Greek samples while samples from the European part of Turkey possess high flavonoid content, similar to European type propolis. 
A third study by Lagouri et al. was focused on the phenolic composition of Greek propolis extracts and their antioxidant properties (Lagouri et al., 2014). Analysis by HPLC-UV showed that West Macedonia propolis contained the highest amount of phenolic compounds. Amongst them phenolic acids (caffeic acid, caffeic acid phenethylester, ferulic acid, p-coumaric acid) and flavonoids (quercetin, galangin, luteolin, apigenin). The most abundant compounds were caffeic acid (up to $4.2 \mathrm{mg} / \mathrm{g}$ propolis) and galangin (up to $8.5 \mathrm{mg} / \mathrm{g}$ propolis).

\section{Portuguese Propolis}

A Portuguese pioneer group has explored the phenolic profile of propolis collected from various regions of Portugal. In the first report of 2010 an ethanolic extract of propolis was prepared, fractionated by HPLC producing numerous fractions which were subjected to further analyses. The identification of the phenolic compounds was achieved by ESI-MS functioning in negative mode (Falcao et al., 2010).

The HPLC profile at $280 \mathrm{~nm}$ was identical for all samples regardless the location and it was consistent with the relationship between the phenolic profile and the surrounding apiary flora.

Simple phenolic acids eluted at first minutes of the run and were caffeic acid (m/z 179), 3,4 dihydroxy vinyl benzene (m/z 135), p-coumaric acid (m/z 163), ferulic and isoferulic acids $(\mathrm{m} / \mathrm{z}$ 193). Esterified and methylated products of these acids were also encountered. Flavonoids such as dihydroflavonols, flavanones, flavones and flavonols were detected and characterized. Particularly aglycone forms of apigenin, pinobanksin, pinocembrin and chrysin and various esterified derivatives. Based on HPLC-UV profile the authors possibly suggested that Portuguese propolis was mostly rich in pinocembrin, chrysin and pinobanksin 3-O-acetate. It should be noted however that the typical flavone galangin was not found in the samples may indicate a peculiar characteristic of the Northeast Portuguese propolis.

Overall, this approach allowed the identification of 37 phenolic compounds. The compounds identified not only belonged to the typical phenolic acids and flavonoids found in propolis from temperate zones but also numerous compounds that have never been reported in literature for propolis matrix.

The same group after three years published a continuation of their early work reporting again on the phenolic profile of Portuguese propolis(Falcao et al., 2013). In this context 40 ethanolic extracts of propolis have been analyzed by HPLC-DAD-ESI-MS ${ }^{n}$ and seventy six polyphenols were detected. Two types of propolis were defined. The first was temperate propolis which contained typical poplar phenolic compounds such as flavonoids and their methylated/esterified forms, phenylpropanoic acids and their esters.

The second type propolis was an uncommon type which contained quercetin and kaempferol glycosides, some of them never described in propolis. The data suggested that other botanical species in addition to poplar trees can be important sources of resins for Portuguese propolis. 


\section{Spanish propolis}

Kumazawa et al. has published a work on the analysis of ethanolic extracts of Spanish propolis originating from Andalusia(Kumazawa et al., 2013). Analysis of samples was performed with a LC-PDA-MS after separating compounds on a C18 column. MS was performed on a LCQ ion trap mass spectrometer operating in the negative ionization mode. LOD and LOQwere determined as the lowest spiking level at which the phenolic compound was consistently identified with a signal to noise ration $\mathrm{S} / \mathrm{N} \geq 3$ and $\mathrm{S} / \mathrm{N} \geq 10$ respectively. An external standard calibration curve was established using five data points. LOD and LOQs ranged from 1.3 to 4.3 and from 3.8 to $12.4 \mu \mathrm{g} / \mathrm{mL}$ respectively; the lowest being observed for caffeic acid.

The most abundant phenolic compounds were flavonoids such as pinobanskin 3-acetate, pinocembrin, chrysin, galangin and pinobanskin. Caffeic acid phenethyl ester was detected in almost all extracts but in smaller proportions compared to flavonoids. The high scavenging activity that these samples exhibited indicates that Andalusian propolis could potentially be used as a rich natural source of anti-oxidants.

\section{Finish propolis}

Salonen et al. have published work on the profile of phenolic compounds after analyzing 19 propolis samples from different provinces in Finland (Salonen et al., 2012). Nine individual flavonoids (26\% of the identified phenolics), eleven cinnamic acid derivatives (36\%), two caffeic acid derivatives (14\%), three chlorogenic acid derivatives (14\%) and three other phenolic acids $(10 \%)$ were found in propolis samples.

The extraction of propolis samples was conducted with methanol. After filtration and centrifugation of $1 \mathrm{~mL}$ of the extract -mixed with $0.5 \mathrm{~mL}$ of water- the supernatant was directly injected to the HPLC system. Separation of compounds was performed on a Zorbax, SB-C18 column using as mobile phase a gradient system consisting of water containing tetrahydrofuran (1.5\%) and orthophosphoric acid (0.25\%) (A) and methanol (100\%).After analyses evaluation, methyl-naringenin, CAPE, a hydroxyl cinnamic acid derivative and benzoic acids were found in the largest quantities. The overall results indicated that no Finish tree species can be pointed out as the sole source for Finnish propolis.

\section{CONCLUSION (FUTURE INSIGHTS)}

Propolis is and will be a constant field of research among scientists from chemistry to medicine, due to its proven beneficial effects and plethora of compounds encompassed in its portfolio. From chemical point of view its extraction seems quite standardized based on organic solvent abstraction of active compounds from powdered material, followed by filtration and centrifugation steps. The extraction time varies from hours to one to two days. The identification of non-volatile compounds is achieved mainly by TLC and HPLC coupled with DAD and MS in simple or tandem mode. Indispensable is the contribution of NMR techniques in structure elucidation of compounds, especially unknown compounds that are isolated either from preparative TLC, column chromatography or preparative HPLC fractionation. 
Judging from existing literature, the analyses of propolis samples -even from regions where detailed studies have been conducted- should be continued as new compounds are likely to be discovered bearing pharmaceutical potential. Emerging analytical techniques utilizing cutting edge mass detectors and other chromatography columns (apart for typical C18 columns) might be regarded in the frames of optimization of separation capacity which is critical for such a complex matrix, as propolis is.

\section{REFERENCES}

1. Aguero, M.B., Gonzalez, M., Lima, B., Svetaz, L., Sanchez, M., Zacchino, S., Feresin, G.E., SchmedaHirschmann, G., Palermo, J., Wunderlin, D., Tapia, A., 2010. Argentinean propolis from Zuccagnia punctata Cav. (Caesalpinieae) exudates: phytochemical characterization and antifungal activity. J Agric Food Chem 58, 194-201.

2. Athikomkulchai, S., Awale, S., Ruangrungsi, N., Ruchirawat, S., Kadota, S., 2013. Chemical constituents of Thai propolis. Fitoterapia 88C, 96-100.

3. Bankova, V., 2005a. Chemical diversity of propolis and the problem of standardization. Journal of ethnopharmacology 100, 114-117.

4. Bankova, V., 2005b. Recent trends and important developments in propolis research. Evidence-based complementary and alternative medicine : eCAM 2, 29-32.

5. Boufadi, Y.M., Soubhye, J., Riazi, A., Rousseau, A., Vanhaeverbeek, M., Neve, J., Boudjeltia, K.Z., Van Antwerpen, P., 2014. Characterization and antioxidant properties of six Algerian propolis extracts: ethyl acetate extracts inhibit myeloperoxidase activity. International journal of molecular sciences 15, 2327-2345.

6. Celemli, O., Hatjina, F., Charistos, L., Schiesser, A., Ozkirim, A., 2013. More Insight into the Chemical Composition of Greek Propolis; Differences and Similarities with Turkish Propolis. Z. Naturforsch. 68c, 429438.

7. Falcao, S.I., Vale, N., Gomes, P., Domingues, M.R., Freire, C., Cardoso, S.M., Vilas-Boas, M., 2013. Phenolic profiling of Portuguese propolis by LC-MS spectrometry: uncommon propolis rich in flavonoid glycosides. Phytochemical analysis : PCA 24, 309-318.

8. Falcao, S.I., Vilas-Boas, M., Estevinho, L.M., Barros, C., Domingues, M.R., Cardoso, S.M., 2010. Phenolic characterization of Northeast Portuguese propolis: usual and unusual compounds. Analytical and bioanalytical chemistry 396, 887-897.

9. ICH, 2005. VALIDATION OF ANALYTICAL PROCEDURES: TEXT AND METHODOLOGY Q2(R1)http://www.ich.org/fileadmin/Public_Web_Site/ICH_Products/Guidelines/Quality/Q2_R1/Step4/Q2_R1 _Guideline.pdf.

10. Kalogeropoulos, N., Konteles, S.J., Troullidou, E., Mourtzinos, I., Karathanos, V.T., 2009. Chemical composition, antioxidant activity and antimicrobial properties of propolis extracts from Greece and Cyprus. Food Chem 116, 452-461.

11. Kumazawa, S., Bonvehi, J.S., Torres, C., Mok-Ryeon, A., Bermejo, F.J., 2013. Chemical and functional characterisation of propolis collected from East Andalusia (southern Spain). Phytochemical analysis : PCA 24, 608-615.

12. Kunimasa, K., Ahn, M.R., Kobayashi, T., Eguchi, R., Kumazawa, S., Fujimori, Y., Nakano, T., Nakayama, T., Kaji, K., Ohta, T., 2011. Brazilian Propolis Suppresses Angiogenesis by Inducing Apoptosis in Tube-Forming Endothelial Cells through Inactivation of Survival Signal ERK1/2. Evidence-based complementary and alternative medicine : eCAM 2011, 870753.

13. Lagouri, V., Prasianaki, D., Krysta, F., 2014. Antioxidant Properties and Phenolic Composition of Greek Propolis Extracts. Int J Food Prop 17, 511-522.

14. Lofty, M., 2006. Biological Activity of Bee Propolis in Health and Disease. Asian Pacific Journal of Cancer Prevention 7, 22-31.

15. Lopez, B.G., Schmidt, E.M., Eberlin, M.N., Sawaya, A.C., 2014. Phytochemical markers of different types of red propolis. Food Chem 146, 174-180.

16. Marquez Hernandez, I., Cuesta-Rubio, O., Campo Fernandez, M., Rosado Perez, A., Montes de Oca Porto, R., Piccinelli, A.L., Rastrelli, L., 2010. Studies on the constituents of yellow Cuban propolis: GC-MS determination of triterpenoids and flavonoids. J Agric Food Chem 58, 4725-4730.

17. Melliou, E., Stratis, E., Chinou, I., 2007. Volatile constituents of propolis from various regions of Greece Antimicrobial activity. Food Chem 103, 375-380.

18. Papotti, G., Bertelli, D., Bortolotti, L., Plessi, M., 2012. Chemical and functional characterization of Italian propolis obtained by different harvesting methods. J Agric Food Chem 60, 2852-2862.

19. Pellati, F., Prencipe, F.P., Bertelli, D., Benvenuti, S., 2013. An efficient chemical analysis of phenolic acids and flavonoids in raw propolis by microwave-assisted extraction combined with high-performance liquid chromatography using the fused-core technology. Journal of pharmaceutical and biomedical analysis 81-82, 126-132. 
20. Petrova, A., Popova, M., Kuzmanova, C., Tsvetkova, I., Naydenski, H., Muli, E., Bankova, V., 2010. New biologically active compounds from Kenyan propolis. Fitoterapia 81, 509-514.

21. Piccinelli, A.L., Mencherini, T., Celano, R., Mouhoubi, Z., Tamendjari, A., Aquino, R.P., Rastrelli, L., 2013. Chemical Composition and Antioxidant Activity of Algerian Propolis. J Agr Food Chem 61, 5080-5088.

22. Popova, M., Chen, C.N., Chen, P.Y., Huang, C.Y., Bankova, V., 2010. A Validated Spectrophotometric Method for Quantification of Prenylated Flavanones in Pacific Propolis from Taiwan. Phytochem Analysis 21, 186-191.

23. Popova, M., Trusheva, B., Antonova, D., Cutajar, S., Mifsud, D., Farrugia, C., Tsvetkova, I., Najdenski, H., Bankova, V., 2011. The specific chemical profile of Mediterranean propolis from Malta. Food Chem 126, 14311435.

24. Pratsinis, H., Kletsas, D., Melliou, E., Chinou, I., 2010. Antiproliferative Activity of Greek Propolis. J Med Food 13, 286-290.

25. Salonen, A., Saarnio, S., Julkunen-Tiitto, R., 2012. Phenolic Compounds of Propolis from the Boreal Coniferous Zone. J Apic Sci 56, 13-22.

26. Segueni, N., Zellagui, A., Moussaoui, F., Lahouel, M., Rhouati, S., Flavonoids from Algerina propolis. Arabian Journal of Chemistry, http://dx.doi.org/10.1016/j.arabjc.2011.1005.1013.

27. Shi, H., Yang, H., Zhang, X., Yu, L.L., 2012. Identification and quantification of phytochemical composition and anti-inflammatory and radical scavenging properties of methanolic extracts of Chinese propolis. J Agric Food Chem 60, 12403-12410.

28. Shirataki, Y., Motohashi, N., Tani, S., Sakagami, H., Satoh, K., Nakashima, H., Mahapatra, S.K., Ganguly, K., Dastidar, S.G., Chakrabarty, A.N., 2001. In vitro biological activity of prenylflavanones. Anticancer research 21, 275-280.

29. Varoni, E.M., Lodi, G., Sardella, A., Carrassi, A., Iriti, M., 2012. Plant polyphenols and oral health: old phytochemicals for new fields. Current medicinal chemistry 19, 1706-1720.

30. Zizic, J.B., Vukovic, N.L., Jadranin, M.B., Andelkovic, B.D., Tesevic, V.V., Kacaniova, M.M., Sukdolak, S.B., Markovic, S.D., 2013. Chemical composition, cytotoxic and antioxidative activities of ethanolic extracts of propolis on HCT-116 cell line. Journal of the science of food and agriculture 93, 3001-3009.

Konstantinos M. Kasiotis Propolis non-volatile constituents: A Review Hygeia.J.D.Med 2014; 6(1):111-121. Available from http://www.hygeiajournal.com / Article ID- Hygeia.J.D.Med/128/14. D.O.I: 10.15254/H.J.D.Med.6.2014.128. 